\title{
An Evaluation of Clinical Decision Support and Use of Machine Learning to Reduce Alert Fatigue
}

\author{
Noura Khreis ${ }^{1}$, Adela S. M. Lau ${ }^{1}{ }^{*}$, Ahmed Al-jedai ${ }^{2}$, Salma M. Al-Khani², Ezdehar H. Alruwaili \\ ${ }^{1}$ School of Business, Madonna University, Livonia, MI 48150, USA. \\ ${ }^{2}$ King Faisal Specialist Hospital \& Research Center, Zahrawi St, Al Maather Riyadh 12713, Saudi Arabia. \\ * Corresponding author. Tel.: +1 734-793-2451; email: slau@madonna.edu \\ Manuscript submitted October 9, 2018; accepted November 19, 2018. \\ doi: 10.17706/ijcce.2019.8.1.32-39
}

\begin{abstract}
Therapeutic duplication alert is one of the Clinical Decision Support Systems (CDSS) that was implemented to help physicians and other healthcare providers in making clinical judgements about the patients' management of therapy and decreasing medication errors. However, there were high override rates of these alerts by physicians as they were deemed to be of non-clinical significance. The quantity of the alerts fired by the system was high leading to "alert fatigue". Thus, the hospital administrators reached an agreement to deactivate it. To assess the validity of this decision, the aim of the study was to analyze the impact of therapeutic duplication alert deactivation on medication errors' rate. This study retrospectively screened a total of 593 electronic Medication Administration Records (e- MAR) of hospitalized patients with 297 e-MARs in the pre-therapeutic duplication alert deactivation period and 296 e-MARs in the post-therapeutic duplication alert deactivation period in a tertiary care hospital in Saudi Arabia. The number and type of duplicate medication errors in each period was documented to determine whether there was a significant difference between the two periods. The results detected 51 out of 297 e-MARs with medication errors in the pre-therapeutic duplication alert deactivation period versus 47 out of 296 in the post alert deactivation therapeutic duplication. Chi square test showed that there was no significant difference in the incidence of medication errors detected among the two periods with a p-value of 0.672 . Therefore, we concluded that there was no significant difference on the medication error after the therapeutic duplication alert deactivation. An integration of machine learning into the clinical decision support design was recommended to filter the duplicated and unimportant alerts and reduce the alert fatigue of physicians.
\end{abstract}

Key words: Clinical decision support, deactivating therapeutic duplication alert, medication error, machine learning.

\section{Introduction}

Clinical Decision Support Systems (CDSS) have used for many years to reduce the medical errors [1]. Medication error is the most common type of medical errors. According to the National Data, on average, each hospitalized patient is subjected to at least one medication error daily. The economic impact of preventable medication errors is huge with total cost of at least $\$ 3.5$ billion yearly [2]. CDSS generated alerts help physicians and other health care professionals to make patients' specific judgments by warning them about possible drug-drug interaction, therapeutic duplication, serious adverse drug reaction including possible allergies and contraindication alerts [3]. 
Several studies, however, have demonstrated that certain CDSS implementations did not achieve the leading-edge advantage that was claimed to do. Alert override rates or non-adherence were high in most hospitals ranging between 49\%-96\% [4]. The reasons behind the high override rates were still unclear, however, some analysts believed that the continuous firing of high quantity of CDSS alerts has caused a phenomenon called "alert fatigue" [5]. As defined by Sijs, alert fatigue was "a mental state that is the result of alerts consuming too much time and mental energy, which can cause relevant alerts to be unjustifiably overridden along with clinically unimportant ones." Since CDSS generated a huge number of clinical alerts, the internal reports from the pharmacy informatics and automation section indicated that the percentage of overridden alerts by physicians and pharmacists exceeded 97\%, and that the most overridden alerts were "therapeutic duplication" alerts. As a result, the morbidity and mortality unit at the hospital recognized the problem and recommended turning off the therapeutic duplication alert system, as it was deemed less important than others.

Therefore, the aim of this study was to investigate whether there is an impact of deactivating therapeutic duplication alert on the number of duplicate medication errors. The solution of reducing the medication errors in clinical decision support will also be presented and discussed in this study.

\section{Literature Review}

The effectiveness of the CDSS became a question to the healthcare institutions and policy makers. Regarding the survey from Wetterneck [6], it showed that the rate of duplicate medication errors before and after the implementation of CDSS did not improve. The study collected 4147 patients' data before implementation of the system and 4013 patients' data post implementation. Surprisingly, the rate of duplicate orders post-implementation was higher than pre-implementation (8.1\%- post versus 2.6\%- pre, with $\mathrm{p}$ value $<0.0001)$. The physicians were confused with the alerts' content, and they believed that the CDSS algorithms were missing true duplicates. Another systemic review was done by Keasberry [7], it also found that there was a conflicting evidence regarding the effect on mortality, medication errors and healthcare cost. CDSS increased alert fatigue, technology-interaction time and even medication errors since the alerts led to continuous interruption of workflow.

Regarding the review study on the advantages of CDSS in improving patient safety and decreasing medication errors [4], it showed that the over-ridden rates of these alerts were as high as $96 \%$ and the authors proposed to improve CDSS alerts responsiveness and decrease alert fatigue by developing innovative methods to evaluate retrospectively which alerts are clinically significant and which ones are not. The study also addressed the utilization of traditional health informatics approaches to customize the challenges in alert evaluations. The bottom line is to improve CDSS to increase patient safety and reduce alert fatigue.

A time series prospective study was done by Magid [8] to investigate the effects of the strategies taken to minimize duplicate orders in a hospital implementing a COPE/CDS system in NewYork city. After over 84 weeks' study, the investigators recognized several factors leading to duplicate orders such as granting access to several physicians to prescribe medications to the same patient. Other reasons included technical issues with CDSS system such as high false positive alerts, and the incorporation of defaulted diagnosis specific care sets. After strategically limiting these factors, the incidence of duplicate orders was minimized significantly from week 1 to week 84 with a rate of $84.4 \%(p<0.001)$.

Regarding another prospective study of 286 adult patients in a tertiary care hospital, the study found that that pharmacists' medication reconciliation was associated with lower medication-related errors compared to physicians' driven medication reconciliation. 3085 medications were identified by clinical pharmacists upon reconciliation. The presence of pharmacists can reduce the medication error rate. Therefore, 
integrating pharmacists' knowledge into a CDSS platform to perform medication alerts decision marking is an alternative approach to reduce the medication alerts error generated by the system.

To capture the physicians' knowledge into the CDSS, machine learning is the current advanced method in computer science study for capturing human knowledge into the system. Machine learning uses artificial intelligence and statistical models to gain the error feedbacks of the system for model improvement. The machine learning process includes a closed feedback loop of data analysis, error evaluation and feedback, model improvement and data re-analysis. This process will iterate until the best model was found. In other words, machine learning has a learning capability. It compares the actual data with the calculated data of the decision model and make necessary adjustment on the weighting of the parameters in the model for accurate improvement. The machine learning model continues to train until the error rate was satisfied. Therefore, by integrating the machine learning algorithms into the CDSS, it can capture the physician' alert selection decision and perform human and machine decision comparison. The errors between the physicians' decisions and the CDSS recommendations can be used in CDSS model improvement. After a certain period of learning on the physicians' decision making by the CDSS, the CDSS's decision model can be improved and become more accurate on medication alerts, thus reducing the alert fatigue effect [9].

\section{Research Method}

Therefore, in this study, we evaluated the medication errors rate on with or without the CDSS. This study randomly selected data from a 1000 bed tertiary hospital in Saudi Arabia. The primary outcome was to measure the number of medication duplication events before and after the deactivation of CDSS. We screened the e-MAR of admitted patients who fulfilled the inclusion criteria over a period of two months prior and after alert deactivation keeping three months' gap in between data collection to avoid selection bias.

A waiver of informed consent was enacted from the King Faisal Specialist Hospital and Research Center's institutional Review Board for retrieval and use of de-identified data, as the study didn't involve manipulation of human health management and no utilization of sensitive patients' data. The following data were extracted from the patients' e-MARs: patients' baseline information such as Medical Record Number (MRN) and medication. The Medial Record Number was automatically given a code for confidentiality before the extraction. No collected data was sensitive. The therapeutic duplication detected was divided in to three categories: an identical order, same medication but different dosage form/frequency/route, and different medication but same therapeutic class.

Descriptive statistics for the continuous variables were reported as mean \pm standard deviation and categorical variables were summarized as frequencies and percentages. The statistical tests used was Chi-square test goodness of fit. The level of statistical significance is set at $\mathrm{p}<0.05$.

\section{Hypothesis Testing}

\section{Effect of Alert Deactivation Decision on Therapeutic Duplication Medication Errors:}

Since the objective of this study is to test whether the deactivation of the therapeutic duplication alert has no effect spread on the number of medications error in the two periods, Chi Square test for Independence and goodness of fit were used to test the following hypothesis:

$\mathrm{H}_{0}$ : Therapeutic duplication medication errors are independent upon alert deactivation decision

$\mathrm{H}_{1}$ : Therapeutic duplication medication errors are dependent upon alert deactivation decision

\section{Results}

A total of 593 e-MARs were scanned for therapeutic duplications with 297 e-MARS in the pre-therapeutic 
duplication period and 296 e-MARS in the post-therapeutic duplication period. 51 (17.17\%) duplicate errors were detected in the first period versus 47 (15.9\%) duplicate errors in the post period. When stratified by Therapeutic Duplication (TD) type, the most common TD error in both groups was prescribing the "same exact medication", followed by giving "same medication with different dosage form/dose", and "same therapeutic category" lastly.

The Chi-Square test was used to test whether the observed difference in the duplications among the two periods is significantly different than the expected data (Table 1-3). The level of significance $(\alpha)$ is set to 0.05. The analysis showed than $X^{2}$ value was 0.179 which is less than $X^{2}$ critical value of 3.841 and $p$-value was $0.672(>0.05)$. Thus, the null hypothesis was rejected. And we can conclude that there is no significant difference between the observed and expected frequencies. The action of deactivating the therapeutic duplication alert has no effect on medication error, and the number of medication errors are spread evenly in the two periods studied.

Table 1. The Observed Duplicate Error

\begin{tabular}{llll}
\hline \hline $\begin{array}{l}\text { Observed/Duplica } \\
\text { te Error }\end{array}$ & $\begin{array}{l}\text { Pre-Period } \\
\text { N (\%) }\end{array}$ & $\begin{array}{l}\text { Post-Period } \\
\text { N (\%) }\end{array}$ & $\begin{array}{l}\text { Grand } \\
\text { Totalc }\end{array}$ \\
\hline No & $246(82.8)$ & $249(84.12)$ & 495 \\
\hline Yes & $51(17.17)$ & $47(15.88)$ & 98 \\
\hline Total & $\mathbf{2 9 7}$ & $\mathbf{2 9 6}$ & $\mathbf{5 9 3}$ \\
\hline \hline
\end{tabular}

Table 2. The Expected Duplicate Error

\begin{tabular}{llll}
\hline \hline $\begin{array}{l}\text { Expected/Duplica } \\
\text { te Error }\end{array}$ & $\begin{array}{l}\text { Pre-Period } \\
\text { N (\%) }\end{array}$ & $\begin{array}{l}\text { Post-Period } \\
\text { N (\%) }\end{array}$ & $\begin{array}{l}\text { Grand } \\
\text { Totalc }\end{array}$ \\
\hline No & $248(83.5)$ & $247(83.4)$ & 495 \\
\hline Yes & $49(16.5)$ & $49(16.6)$ & 98 \\
\hline Total & $\mathbf{2 9 7}$ & $\mathbf{2 9 6}$ & $\mathbf{5 9 3}$ \\
\hline \hline
\end{tabular}

Table 3. Chi-Square Test

\begin{tabular}{|c|c|c|c|}
\hline Observed & Expected & $(O-E)^{2}$ & $(O-E)^{2} / E$ \\
\hline 246 & 248 & 3.676 & 0.015 \\
\hline 249 & 247 & 3.676 & 0.015 \\
\hline 51 & 49 & 3.676 & 0.015 \\
\hline 47 & 49 & 3.676 & 0.015 \\
\hline \multicolumn{2}{|c|}{ df: 1} & $x^{2}$ & 0.180 \\
\hline \multicolumn{2}{|c|}{ Critical Value: 3.841} & p-value & 0.672 \\
\hline
\end{tabular}

\section{Discussion}

From the results of the hypothesis testing, we found that there was no significant difference between with or without CDSS for therapeutic medication alerts. One of the major reasons of this phenomenon was due to the alert fatigue. As the volume of the alerts fired from the CDSS is huge and not all with clinical importance, as a result, it causes the physicians to ignore almost the alerts [6]. Some authors proposed to provide a tailored or parsimonious warning in CDSS design that could reduce alert fatigue [10]. However, how the CDSS decision rules being able to be adjusted based on different clinical situations is another question. Some other researchers suggested to develop a framework for evaluating the types and importance of alerts that could reduce the duplication or huge volume of alerts to physicians [11], [12]. Heringa [13] proposed to develop a specification of triggers in CDSS design to classify the types of alerts and the degree of importance. However, the classification model for the alerts may vary based on patients' clinical situation and depends on the physicians' clinical experience to translate how important medical alerts to action. We cannot pre-define the rules for classifying the importance of medical alerts. The decision rules should be able to 
adaptive to environment for changes based on the clinical situations. Therefore, in the study of Coleman [14], it proposed to design CDSS with some intelligence for determining the optimal sensitivity and specificity of alerts; being able to adapt to the environment or characteristics of the user and modify the timing and number of alerts.

However, since machine learning has the learning capability, it can learn the historical lessons and make changes of the decision rules based on the scenario environment's changes [15]. Integrating machine learning into the clinical decision support design allows the CDSS to learn the decision behavior of the physicians and make necessary adjustment on the rules in CDSS's rule-based engine and filter the duplicated and unimportant alerts in adaptive environment that reduce the alert fatigue of physicians [16]. Some studies used machine learning methods such as prediction-based threshold [17] and probabilistic model [9] successfully reducing the medication alert errors and made adaptive change on the rules based on the environmental changes. Therefore, by capturing physicians' knowledge can dynamically improve the decision rules of CDSS based on the clinical situation.

\section{Recommendation}

Therefore, we proposed to integrate the machine learning into the CDSS design to learn the physicians' behavior on alert selection and decision making. The following Fig. 1 is the system overview to illustrate the preliminary idea of the machine learning.

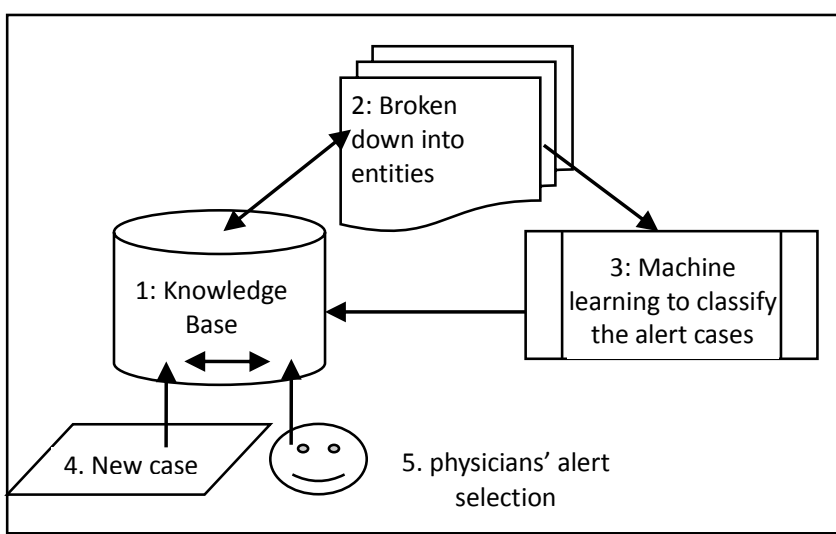

Fig. 1. An overview of integrate the machine learning into the CDSS design.

Step 1: An alert knowledge base and learning interface were implemented to capture all the decision cases of physicians on alerts selection.

Step 2: Each captured case in the knowledge base was broken down into different entities including clinical diagnosis, number of physicians participating in the clinical diagnosis, types of drugs prescribed, prescribing volume, frequency of drugs intake, and patients' demographic, allergy and drug interaction records.

Step 3: A machine learning model was developed and used to learn the physicians' alerts selection decision process. The machine learning algorithm correlated the type of alerts selected and the entries' values in each case. Another machine learning algorithm of similarity measurement was used to group and classify the similar cases that have the similar alerts selected by the physicians for action. The captured similar cases associated with the type of alerts was stored as a new rule for alert selection and was indexed with a scenario number in the rule-based engine.

Step 4: When a new case comes, the case will be broken down into entities. Its corresponding values will be captured and stored in it. A machine learning of similarity measurement will be applied to compare the similarity of the new case with the cases in knowledge base. Once, it was mapped into a category of the case, 
the alert selection rules of that case category will be used to determine which alerts to be showed to the CDSS interface.

Step 5: If the CDSS alerts was not selected by the physician, the errors of alerts selection will be fed into the rule-based engine to re-train the model by using the machine learning algorithm.

Since the alert selection rule-based engine was well-trained with the previous cases, the accuracy of the alert selection by CDSS will be improved and it will reduce the alert fatigue behavior of the physicians.

\section{Conclusion}

The study showed that the decision to deactivate therapeutic duplication medication error alert didn't have any significant impact on the incidence of medication errors in hospitalized patients. This phenomenon showed that the computerized decision support system may not be able to reduce the medical errors as the original system design goals. Integrating machine learning into the clinical decision support design allows CDSS to learn the physicians' decision process that can reduce the fault medication alerts. Machine learning captures and learns the decision process and filter the duplicated alerts in various scenarios that ultimately can reduce the alert fatigue of physicians.

\section{Acknowledgment}

We would like to acknowledge the Research Ethics Committee (REC) of the research advisory council of King Faisal Specialist Hospital and Research Center to approve the first author to access and use the de-identified data for analysis in this project (project no.: RAC \#2131 100). We would also like to acknowledge School of Business of Madonna University to provide the course platform of MIS 5210 for the authors to work on this project.

\section{References}

[1] Jha, A. K., DesRoches, C. M., Campbell, E. G., Donelan, K., Rao, S. R., Ferris , T. G., Shields, A., Rosenbaum, S., \& Blumenthal, D. (2009). Use of electronic health records in U.S. hospitals. The New England Journal of Medicine, 360(16), 1628-1638.

[2] Silva, B. A., \& Krishnamurthy, M. (2016). The alarming reality of medication error: A patient case and review of Pennsylvania and national data. Journal of Community Hospital Internal Medicine Perspectives, 6(4), 31758.

[3] Jia, P., Zhang, L., Chen, J., Zhao, P., \& Zhang, M. (2016). The effects of clinical decision support systems on medication safety: An overview. PLoS One, 11(12), e0167683.

[4] McCoy, A., Thomas, B., Eric, J., Krousel-Wood, M., \& Sittig, D. F. (2014). Clinical decision supports alert appropriateness: A review and proposal for improvement. The Ochsner Journal, 14(2), 195-202.

[5] Sijs, H. (2009). Drug safety alerting in computerized physician order entry: Unraveling and $\begin{array}{llll}\text { counteracting } & \text { alert } & \text { fatigue. } & \text { Retrieved }\end{array}$ https://www.researchgate.net/publication/241860650_Drug_safety_alerting_in_computerized_physici an_order_entry_Unraveling_and_counteracting_alert_fatigue

[6] Wetterneck, T., Walker, J., Blosky, M., Cartmill, R., Hoonakker, P., Johnson, M., Norfolk, E., \& Carayon, P. (2011). Factors contributing to an increase in duplicate medication order errors after CPOE implementation. J Am Med Inform Assoc., 18, 774-782.

[7] Keasberry, J., Scott, I. A., Sullivan, C., Andrew, M., \& Ashby, R. (2017). Going digital: A narrative overview of the clinical and organizational impacts of eHealth technologies in hospital practice. Australian Health Review, 41(6), 646-66.

[8] Magid, S., Shaha, S., \& Forre, C. (2012). Duplicate orders: An unintended consequence of computerized 
provider/physician order entry (CPOE) implementation. Applied Clinical Informatics, 3(4), 377-391.

[9] Nguyen, P. A., Abdul, S. S., Iqbal, U., Hsu, M. H., Huang, C. L., Li, H. C., Clinciu, D. L., Jian, W. S., \& Li, Y. C. J. (2013). A probabilistic model for reducing medication errors. PLOS One, 8(12), e82401.

[10] Kesselheim, A. S., Cresswell, Phansalkar, K. S., Bates, D. W., \& Sheikh, A. (2011). Clinical decision support systems could be modified to reduce 'alert fatigue' while still minimizing the risk of litigation. Health Affairs, 30(12), 2310-2317.

[11] McCoy, A. B., Waitman, L. R., Lewis, J. B., Wright, J. A., Choma, D. P., Miller, R. A., \& Peterson, J. F. (2012). A framework for evaluating the appropriateness of clinical decision support alerts and responses. Journal of the American Medical Informatics Association, 19(3), 346-352.

[12] Ancker, J. S., Kern, L. M., Abramson, E., \& Kaushal, R. (2012). The triangle model for evaluating the effect of health information technology on healthcare quality and safety. Journal of the American Medical Informatics Association, 19(1), 61-65.

[13] Heringa, M., Heide, A., Floor, A., Schreudering, D., Smet, P., \& Bouvy, M. L. (2018). Better specification of triggers to reduce the number of drug interaction alerts in primary care. International Journal Medical Informatics, 109, 96-102.

[14] Coleman, J. J., Sijs, H., Haefeli, W. E., Slight, S. P., McDowell, S. E, Seidling, H. M., Eiermann, B., Aarts, J., Ammenwerth, E., Ferner, R. E., \& Slee, A. (2013). On the alert: Future priorities for alerts in clinical decision support for computerized physician order entry identified from a European workshop. $B M C$ Medical Informatics and Decision Making, 13, 111-120.

[15] Lau, A., Tsui, E., \& Lee, W. B. (2009). An ontology-based similarity measurement for problem-based case reasoning. Expert systems and Application, 43(3), 6547-6579.

[16] Chen, L., Dubrawski, A., Wang, D., Fiterau, M., Bert, M. G., Bose, E., Kaynar, A. M., Wallace, D. J., Guttendorf, J., Clermont, G., Pinsky, M. R., \& Hravnak, M. (2016) Using supervised machine learning to classify real alerts and artifact in online multi-signal vital sign monitoring data. Critical Care Medicine, 44(7), 456-63.

[17] Kawazoea, Y, Miyoa, K., Kurahashib, I., Sakuraia, R., \& Ohea, K. (2013). Prediction-based threshold for medication alert. MEDINFO, 229-231.

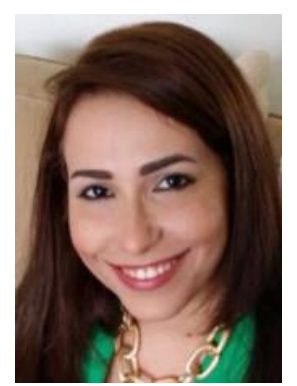

Khreis Noura is a doctor of pharmacy graduate from the Lebanese American University. She is currently pursuing the master's degree in health services administration. She is American Board Certified in Nutrition Support Pharmacy. She was acting the director for the PGY-2 Nutrition Board Pharmacy Specialty Program at KFSHRC. She was assigned as an adjunct clinical preceptor for King Saud University, \& Al-Qassim University.

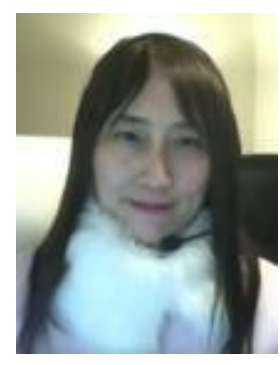

Adela S.M. Lau is an assistant professor of data analytics and business research in the School of Business and teaches health care informatics, IS/technology management, data analytics, and business research. Her research specialties include risk management and big data analytics, e-learning and knowledge management, and e-business strategies, informatics and IS applications in in finance/healthcare/marketing/enterprise. She is currently the editorial board member of the International Journal of Knowledge Engineering and Data Mining and the International Journal of E-Healthcare and is the 
reviewer of several top journals, such as Journal of Medical Internet Research, Industrial Management \& Data Systems, Expert Systems with Applications, etc.

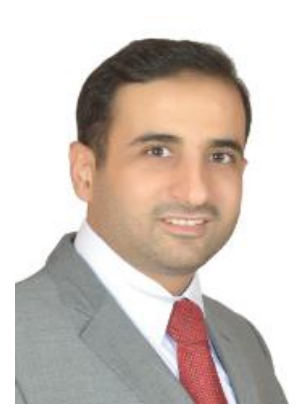

Ahmed Al-jedai is a professor at the Schools of Medicine and Pharmacy at Alfaisal University. He is board certified as Pharmacotherapy Specialist (BCPS), a fellow of the American College of Chest Physicians (FCCP) and the American Society of Transplantation (FAST). He works as the assistant deputy minister for Therapeutic Affairs, Ministry of Health, Saudi Arabia.

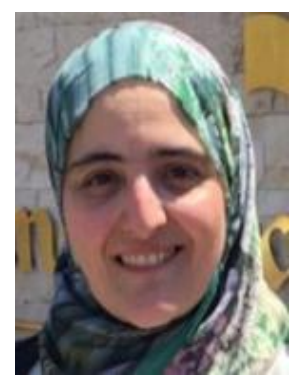

Salma M. Al-Khani is the medication safety officer in King Faisal Specialist Hospital \& Research Center, Riyadh Saudi Arabia. She is with a baccalaureate degree in pharmacy, and a master's degree in health and hospital administration from King Saud University. She is a pharmacy surveyor in the Central Board for Accreditation of Healthcare Institution

Ezdehar H. Alruwaili is the manager of King Abdullah Center of Oncology and liver disease pharmacy in King Faisal Specialist Hospital \& Research Center, Riyadh Saudi Arabia. Ezdehar H. Alruwaili is with a bachelor's degree in pharmacy, and a master degree in health and hospital administration from King Saud University. 